\title{
Vulnerabilidade de adolescentes afrodescencentes e brancos em relação ao HIV/Aids
}

\author{
Vulnerability of teenagers African descending \\ and whites to HIV/Aids
}

\author{
Brigido Vizeu CAMARGO \\ Andréia Isabel GIACOMOZZI² \\ João Fernando Rech WACHELKE \\ Adriana de AGUIAR $^{2}$
}

\begin{abstract}
Resumo
Trata-se de um estudo quantitativo e comparativo entre adolescentes brancos e afrodescendentes sobre vulnerabilidade ao HIV/Aids. A amostra não é randômica e foi formada por 715 estudantes do ensino público noturno de escola de periferia de Santa Catarina. O instrumento utilizado foi um questionário autoadministrado com questões fechadas. Foram analisadas cinco dimensões: afetiva e sexual, conhecimento sobre Aids, atitude, risco e percepção de risco e conduta protetora. Verificou-se que 73,8\% dos adolescentes já tiveram relações sexuais e o namoro é complicador para a prática do sexo seguro. Os brancos obtiveram uma média de 6,42 acertos no subteste de conhecimento sobre Aids contra 6,22 dos afrodescendentes. Ambos os grupos apresentam atitudes favoráveis ao preservativo, porém as meninas apresentam atitude mais favorável do que os meninos. Os brancos consideram-se mais bem informados sobre Aids do que os afrodescendentes. A vulnerabilidade diante do HIV/Aids apresentouse associada à situação sociocultural desfavorável dos adolescentes e não a fatores étnicos.
\end{abstract}

Unitermos: Aids. Atitudes. Adolescentes. Grupos étnicos. HIV.

\begin{abstract}
It is a study which compares white and African descending teenagers on HIV/Aids vulnerability. The sample is not fortuitous, the participants had been 715 students of the nocturnal public education of school of periphery of Santa Catarina. The used instrument was a questionnaire auto-managed with closed questions. Five dimensions were analyzed: sexual and affective, knowledge on Aids, attitude, risk and riskperception and protection behavior. It was verified that $73,8 \%$ of teenagers had already had sexual relations and that a steady relationship is a complicating factor for safe sex practice. White students have obtained a mean of 6,42 scores on the Aids knowledge sub-test against 6,22 from African descending ones. Both groups presented favorable attitudes towards condom use, but girls had more favorable attitudes than boys. Whitestudents considered themselves to be more informed about HIV/Aids than did African descendents. HIV/Aids vulnerability presented itself to be associated to teenagers' unfavorable sociocultural situation and not to ethnic factors.
\end{abstract}

Uniterms: Aids. Attitudes. Teenagers. Ethnic groups. Human immunodeficiency virus.

- Universidade Federal de Santa Catarina, Departamento de Psicologia, Laboratório de Psicossociologia da Comunicação e da Cognição Social. Campus Universitário, Trindade, 88040-970, Florianópolis, SC, Brasil. Correspondência para/Correspondence to: B.V. CAMARGO. E-mail: <brigido.camargo@yahoo.com.br>.

2 Psicólogos. Florianópolis, SC, Brasil.

Apoio: Pesquisa financiada pelo Acordo de cooperação PN-DST/Aids-SUS/Ministério da Saúde/BIRD/UNESCO processo CVs 211/06. 
De acordo com a United Nations Programme on HIV/AIDS (UNAIDS), embora as estimativas globais possam estar mais baixas, os dados continuam a confirmar que a Aids é a principal causa de morte na África. Mais de dois terços das pessoas vivendo com HIV moram na região subsaariana, onde também ocorrem mais de 75\% dos óbitos associados ao HIV. Além disso, quase um terço de todas as novas infecções e de todos os óbitos acontecem em oito países da região sul da África (Botsawa, Lesotho, Moçambique, Namíbia, África do Sul, Swazilândia, Zâmbia e Zimbabwe).

Segundo Bastos, Telles, Castilho e Barcelos (1999), a Aids apresenta taxas de incidência substancialmente mais elevadas nas regiões periféricas e mais pobres, entre os trabalhadores menos qualificados e/ou pessoas com menor grau de escolarização. Houve mudanças na razão homem/mulher entre os casos da doença, com o incremento da participação proporcional das mulheres e pelo estabelecimento de diferenças sociogeográficas no tempo de sobrevida das pessoas com Aids (menor entre as pertencentes às áreas e segmentos mais pobres). Além disso, na dinâmica da epidemia, essas regiões da periferia sobressaem ainda como categorias de exposição à transmissão heterossexual.

Com relação à contaminação de jovens negros pelo HIV/Aids, as estatísticas apontam para uma situação ainda mais grave. Segundo Keels (2005), na Carolina do Norte, a incidência de HIV/Aids é 14 vezes maior entre mulheres afrodescendentes heterossexuais entre $18 \mathrm{e}$ 40 anos do que em mulheres brancas da mesma idade. Ainda de acordo com esse autor, $70 \%$ dos homens jovens com HIV/Aids na Carolina do Norte e nos Estados Unidos são afrodescendentes. No Brasil, segundo dados do Boletim Epidemiológico do Ministério da Saúde (Brasil, 2007), a razão entre brancos e afrodescendentes contaminados pelo HIV está diminuindo, sendo essa diferença mais proeminente em relação ao sexo feminino (de 1,6:1 em 2001 para 1,1:1 em 2006).

Para Batista (2005), as taxas de mortalidade por HIV/Aids no estado de São Paulo em 1999 foram de 25,92 por 100 mil habitantes para homens afrodescendentes, e de 14,44 para brancos. Dentre as mulheres, as taxas são de 11,39 e 4,92, respectivamente para as afrodescendentes e brancas. A razão entre a taxa de mortalidade de mulheres afrodescendentes sobre brancas indica que as primeiras morrem 2,3 vezes mais por HIV/Aids, enquanto que os homens afrodescendentes morrem 1,7 vezes mais que os brancos. Lopes, Buchalla e Ayres (2007), em estudo étnico-racial com mulheres portadoras do HIV no estado de São Paulo, constataram que as mulheres afrodescendentes tinham um status socioeconômico pior do que as brancas e também apresentavam maiores dificuldades para compreender algumas informações sobre HIV/Aids repassadas pelos profissionais de saúde. Além disso, dentre todas as participantes do estudo, as mulheres afrodescendentes tinham menos acesso a outros profissionais de saúde além do médico infectologista, quando comparadas com as mulheres brancas. Os dados mostram que, além de estar "feminilizando, proletarizando e pauperizando", a morte por Aids está "enegrecendo".

Em Santa Catarina, de acordo com Leite (1996, 1998), existe uma "invisibilidade" histórica e política dos afrodescendentes, refletindo uma realidade não muito diferente da do resto do país. Nesse estado, eles estão ocupando os cargos menos remuneráveis nas empresas, estudando em escolas públicas na periferia das cidades e têm as piores condições de acesso à saúde, cultura, informação etc. Além, é claro, de serem constantemente vítimas de preconceito e segregação racial - hoje em dia mais velados -, que ainda marcam o contexto do dia a dia dos cidadãos afrodescendentes. Esses fatores estão relacionados à sua vulnerabilidade específica em relação a essa epidemia.

O conceito de vulnerabilidade é originário da saúde pública e resultou de um processo entre o ativismo diante da epidemia da Aids e o movimento de direitos humanos, bem como da necessidade de um diagnóstico das tendências mundiais da pandemia da Aids no início da década de 1990 (Brasil, 2003). Além disso, esse conceito emergiu a partir das discussões acerca de fator, grupo e comportamento de risco em relação à Aids, mais propriamente das lacunas identificadas nesses discursos (Ayres, França Jr, Calazans \& Saletti Filho, 1999), e teve como objetivo superar a ideia individualista a respeito das determinantes para a prevenção, cujo foco deixa de ser o indivíduo e passa a ser suas relações (Camargo \& Bertoldo, 2006). A vulnerabilidade é entendida como a busca por uma síntese conceitual e prática das dimensões sociais político-institucionais e comportamentais, associadas às dife- 
rentes suscetibilidades de indivíduos, grupos populacionais e até mesmo nações à infecção pelo HIV e às suas consequências indesejáveis (Ayres, Júnior \& Calazans, 1997).

A vulnerabilidade tem ainda ligações com aspectos cognitivos (conhecimento cotidiano da epidemia, as informações sobre ela, as atitudes, a percepção do risco), os sentimentos associados (como o medo), e as condutas relacionadas à doença (intenção de comportamento, atitude diante do preservativo, hábitos e experiência sexuais, comportamentos arriscados). Muito próximo do conceito de vulnerabilidade está o de risco, que se refere mais diretamente à possibilidade de contaminação. Segundo Cochran e Mays (1989, p.8):"O risco da infecção pelo HIV para qualquer indivíduo depende não somente da ocorrência de comportamento de alto risco, mas da performance para este comportamento no meio onde o HIV está presente ... a possibilidade da infecção é influenciada por muitos fatores, incluindo a região geográfica onde o indivíduo reside, o uso ou não de drogas injetáveis, o número de parceiros sexuais, e os tipos e práticas sexuais, entre outros" (Tradução feita pelos autores).

Observa-se na definição de Cochran e Mays (1989) que a maior parte dos fatores que concretizam a ideia de risco envolve comportamentos. Por essa razão, o conceito de atitude é importante para compreendermos a vulnerabilidade diante do HIV/Aids. Segundo Vala (2000), o conceito de atitudes tem ligação com os comportamentos, pois pretende ser mediador entre a forma de pensar e a forma de agir dos indivíduos. As atitudes permitem identificar o posicionamento de um indivíduo diante da realidade social e possuem três características: a) referem-se a experiências subjetivas, b) são sempre referidas a um objeto, e c) incluem uma dimensão avaliativa desse objeto. Para Moscovici (2004), os psicólogos sociais estudam as atitudes como um tipo de substituto do comportamento, seria uma espécie de preparação para o comportamento, que Ihes permitiria predizê-lo para modificá-lo. Assim, estudando as atitudes dos adolescentes frente ao preservativo, por exemplo, seria possível conhecer previamente seu comportamento com relação ao sexo seguro.

O modelo de crença de saúde utilizado nesta pesquisa é o da "Ação Refletida" de Fishbein e Ajzen (1975). De acordo com Stroebe e Stroebe (1995), essa teoria prevê a intenção comportamental e supõe que o comportamento é função dessa intenção. Uma intenção comportamental é determinada pela atitude do sujeito em relação ao desempenho do comportamento e por normas subjetivas. Portanto, as intenções das pessoas com relação ao uso do preservativo, por exemplo, dependerão das suas atitudes com relação ao uso do preservativo, que por sua vez resultam das suas crenças sobre as consequências desse uso ou não uso. A percepção das consequências do uso e do não uso do preservativo pode, portanto, afetar a intenção de usá-lo ou não, mas apenas se os indivíduos acreditarem que as consequências negativas do não uso recaiam sobre si próprios. Assim, por meio deste estudo, pesquisou-se os aspectos interacionais da epidemia da Aids com adolescentes afrodescendentes e não afrodescendentes, considerando sua percepção do risco, atitudes (variáveis afetivas e comportamentais) e o conhecimento sobre a doença. Para este estudo, considerou-se afrodescendentes os jovens que se declararam negros, mulatos e pardos, de acordo com a classificação utilizada na chamada de pesquisa do PN-DST/Aids, por meio da qual este trabalho foi financiado.

\section{Método}

\section{Participantes}

A amostra não é randômica e foi formada por 715 estudantes afrodescendentes e não afrodescendentes do ensino médio, de bairros periféricos, do turno noturno, das cidades de Florianópolis, Balneário Camboriú e Itajaí (Santa Catarina). Os critérios para inclusão na pesquisa foram: morar em uma das três cidades, serem estudantes do ensino médio vinculado às escolas participantes e estar dentro da faixa etária de 15 a 24 anos. Participaram aproximadamente 240 estudantes de cada cidade, sendo metade deles de cada sexo, metade afrodescendente e metade não afrodescendente.

\section{Instrumentos}

Foi utilizado um questionário autoadministrado, composto de questões fechadas e escalas, adaptado a partir de instrumento de Camargo, Botelho e Souza 
(2001). Esse instrumento media conhecimento sobre a Aids, atitudes diante do preservativo e práticas sexuais. As adaptações ocorreram a partir de dados encontrados em estudo anterior (Camargo, Giacomozzi, Wachelke \& Aguiar, 2007), para o qual foram realizadas entrevistas em profundidade com 36 jovens estudantes moradores das mesmas cidades do presente estudo. Dentre as variáveis incluídas no questionário, indicadas pelos resultados, pode-se mencionar as seguintes: nível sociocultural, prática religiosa, comportamentos relativos às relações amorosas, conhecimento sobre doenças sexualmente transmissíveis e exposição à violência. As questões do questionário utilizado neste estudo podem ser classificadas em quatro grupos: 1) características individuais dos participantes (sexo, idade, ambiente social, experiência amorosa e sexual, contexto de comunicação sobre sexualidade); 2) conhecimento ligado ao HIV/Aids ${ }^{3}$ e autopercepção do conhecimento sobre esta epidemia; 3) atitudes sobre o uso do preservativo ${ }^{4}$, percepção e sentimentos em relação ao risco de infecção; 4) comportamentos arriscados e de proteção em relação à Aids.

\section{Procedimentos}

A coleta de dados ocorreu em escolas de Florianópolis, Itajaí e Balneário Camboriú, no período de fevereiro e março de 2007. O estudo foi aprovado pelo Comitê de Ética em Pesquisa com seres humanos da Universidade Federal de Santa Catarina, sob o protocolo 266/2005.

Foram realizadas análises estatísticas descritivas e análises de associação entre as variáveis com o software SPSS (Pacote Estatístico para as Ciências Sociais). Na análise das tendências globais das respostas, utilizou-se da distribuição dos valores conferidos pelas respostas a cada questão que comportava uma medida categorial, e a média e desvio-padrão para questões envolvendo escalas numéricas (análise estatística descritiva). A comparação de subamostras (etnia e sexo) foi executada por meio de tabelas de contingências (teste estatístico não paramétrico - qui-quadrado) e de comparação entre médias (teste de Student e análise de variância simples) para analisar as relações entre os dois grupos étnicos, em função dos quatro grupos de questões (variáveis). Para a escala de atitude, utilizou-se o método da tendência linear ao ponto, na estimação das médias nos itens onde respondentes assinalaram a modalidade "não sei" ou onde eles deixaram em branco, para a obtenção do escore médio.

Realizou-se uma categorização do nível sociocultural dos participantes conforme critério já utilizado por Camargo (1997): foi solicitado aos participantes que indicassem a profissão e o nível de escolaridade de seus pais, e foram considerados como de nível sociocultural Desfavorável os filhos de pais com até ensino médio incompleto, com profissões simples, como operários e pequenos profissionais liberais. Filhos de pais operários, funcionários ou profissionais liberais com pelo menos ensino médio completo foram classificados como tendo nível sociocultural Médio. Filhos de funcionários ou profissionais liberais com ensino superior foram classificados como nível sociocultural Favorável. Foi observado um equilíbrio entre as indicações sobre pais e mães, visando-se chegar a um nível sociocultural entre ambos.

\section{Resultados}

A média (M) das idades dos participantes foi de dezessete anos e três meses e o Desvio-Padrão (DP) de dois anos (Tabela 1).

Os participantes que se declararam negros, pardos e mulatos foram considerados afrodescendentes, totalizando 355 indivíduos (ou 49,7\% do total). A partir da classificação dos participantes quanto ao seu nível sociocultural, observou-se que quatro quintos da amostra apresentaram nível sociocultural desfavorável e houve associação significativa entre a etnia dos participantes (branco ou afrodescendente) e seu nível sociocultural $\left[\chi^{2}=7,21 ; g l=2 ; p<0,05\right]$, o que indica que há mais afrodescendentes com nível sociocultural desfavorável do que brancos e mais brancos com nível sociocultural médio que afrodescendentes.

3 Consiste no sub-teste "O vírus da Aids e sua transmissão", integrante de uma medida sobre conhecimento da Aids construída por Camargo, Barbará e Bertoldo (2005).

4 A atitude dos participantes foi medida por uma escala do tipo Likert com cinco pontos, composta de 12 itens. A consistência entre os itens, em estudo anterior (Camargo et al., 2001), foi moderada ( $\alpha=0,75)$. 
Tabela 1. Distribuição do perfil dos participantes por cidade, série, sexo e etnia.

\begin{tabular}{lcc}
\hline \multirow{2}{*}{ Variável } & \multicolumn{2}{c}{ Participantes } \\
\cline { 2 - 3 } & $\mathrm{n}$ & $\%$ \\
\hline Cidade & 240 & 33,6 \\
Florianópolis & 235 & 32,9 \\
Balneário Camboriú & 240 & 33,6 \\
Itajaí & & \\
Série & 283 & 39,6 \\
$1^{\circ}$ ano & 184 & 25,7 \\
$2^{\circ}$ ano & 248 & 34,7 \\
$3^{\circ}$ ano & & \\
Sexo & & \\
Masculino & 367 & 51,3 \\
Feminino & 348 & 48,7 \\
Etnia & & \\
Branco & 360 & 50,3 \\
Negro & 90 & 12,6 \\
Mulato & 135 & 18,9 \\
Pardo & 130 & \\
\hline
\end{tabular}

\section{Experiências afetivas e sexuais}

Pouco mais de dois terços dos participantes disseram que costumam "ficar", e uma maior proporção de afrodescendentes relataram ter esse tipo de comportamento: $73,9 \%\left[\chi^{2}=8,61 ; \mathrm{gl}=1 ; \mathrm{p}<0,01\right]$. A maior parte dos participantes (59\%) disse não namorar no momento da pesquisa, porém mais brancos do que afrodescendentes declararam ter namorado (73,9\% contra 63,3\%). Contudo, não houve diferença significativa nas proporções $\left[\chi^{2}=2,05 ; g l=2 ; N S\right]$.

A maioria dos respondentes $(73,7 \%)$ relatou que já teve pelo menos uma relação sexual com penetração. Os adolescentes brancos foram mais numerosos que os afrodescendentes no que se refere a ter relações sexuais com muita frequência (40,5\% contra 36,2\%). Contrariamente, houve mais afrodescendentes que declararam nunca terem tido relações sexuais (28,2\% contra 24,6\% dos brancos), porém essas diferenças também não foram estatisticamente significativas $\left[\chi^{2}=1,77 ; g \mid=2 ; N S\right]$.

Considerando somente os participantes com experiência sexual, 85,2\% disseram ter tido relações sexuais nos doze meses anteriores à coleta de dados. A maioria desse último conjunto de participantes $(61,8 \%)$ declarou que essas relações se deram com o mesmo parceiro. Não houve associação significativa entre ter relações sexuais nos doze meses anteriores à coleta e etnia $\left[\chi^{2}=3,70 ; \mathrm{gl}=2 ; \mathrm{NS}\right]$.

\section{Relações entre experiências afetivas e sexuais}

Dentre os participantes que tinham o costume de ficar, a maior proporção disse ter tido relações sexuais uma ou "algumas vezes" (42,3\%), seguida por aqueles que disseram tê-las experimentado "muitas vezes" (32,5\%). Entre os que relataram não ficar, o padrão se inverte: quase metade disse ter tido relações sexuais com penetração e 20,9\% relataram ter tido relações apenas uma ou duas vezes. Houve associação significativa entre o comportamento de ficar e ter tido relação sexual $\left[\chi^{2}=32,10 ; g l=2 ; p<0,001\right]$.

Ao cruzar os dados referentes aos participantes que já haviam tido relações sexuais sobre o comportamento de ficar e a ocorrência de relações sexuais nos doze meses anteriores à coleta, verificou-se que a maior proporção dos participantes que disseram costumar ficar teve relações sexuais com mais de uma pessoa (32,3\%). Entre os participantes que relataram não ter o costume de ficar, esse percentual cai para (6,5\%), bem como havia mais participantes que não costumavam ficar que tiveram relações sexuais com a mesma pessoa $(87,1 \%)$, comparados com os demais $(50,3 \%)\left[\chi^{2}=62,53\right.$; $g \mathrm{l}=2 p<0,01]$.

\section{Experiências afetivas, sexuais e prática religiosa}

Considerando a declaração de prática religiosa dos participantes, há proporcionalmente mais não praticantes que ficam $(72,8 \%)$ do que praticantes $(61,8 \%)$ $\left[\chi^{2}=7,81 ; \mathrm{gl}=1 ; p<0,01\right]$. Dentre os praticantes, a amostra dividiu-se mais igualitariamente entre os que nunca tiveram relações sexuais, tiveram algumas e tiveram muitas relações, com predomínio dos que nunca tiveram (37,8\%). Entre os não praticantes, os participantes concentraram-se nas modalidades "algumas vezes" (40,3\%) e"muitas vezes" (39,8\%). A prática religiosa apresenta associação estatisticamente significativa com o fato de declarar não ter tido relação sexual com penetração $\left[\chi^{2}=3,12 ; g l=1 ; p<0,05\right]$. Considerando a variável etnia como controle, observou-se que tanto entre os brancos $\left[\chi^{2}=15,87 ; g \mid=2 ; p<0,001\right]$ como entre 
os afrodescendentes $\left[\chi^{2}=12,42 ; g l=2 ; p<0,01\right]$, a prática religiosa está associada à declaração de não ter tido relação sexual com penetração.

Com relação a comparações entre os sexos, foi observada uma maior proporção de rapazes que costumam ficar (78,2\%) em comparação com as moças (58,1\%), associação que foi estatisticamente significativa $\left[\chi^{2}=31,99 ; g \mid=1 ; p<0,001\right]$

\section{O conhecimento da Aids}

Considerando as proporções de participantes que indicaram as fontes de informação como origem da maioria de suas informações, as principais, em ordem decrescente de importância, foram: escola, televisão, família, folhetos informativos, jornais e revistas, profissionais de saúde, e amigos (Figura 1).

\section{Conhecimento}

Com relação ao conhecimento sobre a doença, os participantes brancos tiveram uma média de 6,42 acertos $(D P=1,70)$ no subteste do instrumento já mencionado, dentre dez possíveis. Os afrodescendentes conseguiram uma média de 6,22 ( $\mathrm{DP}=1,92)$. A diferença entre etnias não foi significativa $[t=1,47 ; \mathrm{gl}=701$; NS]. Seis acertos significam estar abaixo da nota de corte de 7 acertos desse subteste.

A respeito de como avaliam a qualidade de seu conhecimento sobre a Aids, metade dos participantes brancos pensam ser bem informados e 23,3\% acreditam

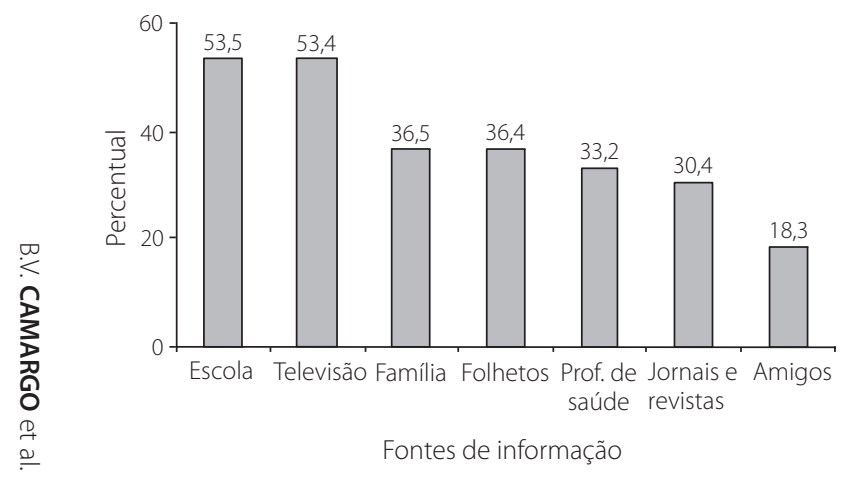

Figura 1. Distribuição das proporções de participantes que indicaram fontes de informação como principais. Florianópolis, Balneário de Camburiú e Itajaí (SC), 2007. não o ser; e para os afrodescendentes, 31,6\% pensam não ser bem informados. Os percentuais de participantes que declararam não saber avaliar a qualidade do seu conhecimento são similares: pouco mais de um quarto do total de cada etnia. Houve associação significativa entre etnia e autopercepção do nível de conhecimento sobre a Aids $\left[\chi^{2}=6,51 ; g l=2 ; p<0,05\right]$.

Foi realizada uma ANOVA $2 \times 3$ com etnia e autopercepção sobre conhecimento sobre Aids. As modalidades da autopercepção foram as seguintes: "pensa que é bem informado", "pensa que não é bem informado" e "não sabe se é bem informado". Essas modalidades foram consideradas fatores de medidas independentes e os escores no teste de conhecimento sobre Aids como variável dependente. Foi encontrado efeito significativo da variável autopercepção sobre conhecimento sobre Aids $[F(2,708)=8,61 ; p<0,001]$. Os participantes que se julgavam mal informados sobre Aids tiveram média de 5,92 acertos ( $D P=1,82)$, os que não sabiam estimar seu grau de informação tiveram média de 6,23 ( $D P=1,70)$, e os que pensavam ser bem informados apresentaram média de 6,61 (DP=1,83). 0 teste de Tukey apontou uma diferença significativa entre as médias dos participantes que pensavam ser bem informados e os participantes que pensavam ser mal informados $(p<0,05)$. Constatou-se certa correspondência entre a autopercepção dos participantes e seu conhecimento sobre Aids: os participantes que pensavam ser mal informados sobre Aids saíram-se pior no subteste, e os que pensavam saber mais obtiveram melhores resultados. Não houve efeitos significativos da variável etnia $[F(1,708)=1,20 ;$ NS] e da interação entre etnia e grau de autopercepção de conhecimento $[\mathrm{F}(2$, 708) $=0,37$;NS].

\section{Atitude em relação ao uso do preservativo}

Comparando-se a atitude dos adolescentes brancos com a dos afrodescendentes acerca do uso do preservativo, foi observado que os primeiros tiveram média de 3,69, numa escala de 1 (atitude desfavorável) a 5 (atitude favorável) e um desvio-padrão de 0,51; e os afrodescendentes tiveram média de 3,65 ( $D P=0,51)$. Comparando-se a atitude por sexo, obteve-se uma média de 3,56 (DP=0,51) para os rapazes, e 3,78 ( $D P=0,49)$ para as moças. Como todas as médias foram superiores 
a 3 (valor do ponto médio), contatou-se que todos os grupos posicionaram-se favoravelmente ao uso do preservativo. Foi realizada uma ANOVA fatorial com sexo e etnia como variáveis independentes e atitude diante do preservativo como variável dependente. Foi encontrado efeito significativo da variável sexo $[F(1,711)=35,94$; $p<0,001]$ : isso se deve ao fato das moças terem apresentado uma atitude ainda mais positiva que a dos rapazes diante do uso do preservativo ( $d=0,44$; efeito médio). Não foi encontrado efeito significativo para a variável etnia $[F(1,711)=0,42$; NS] e para a interação entre etnia e sexo $[F(1,711)=0,03 ; N S]$.

Quanto ao sentimento de medo diante da Aids, compararam-se as médias por etnia num item com forma escalar (variando de 1 a 4, de "nenhum" a "muito medo"). Os brancos tiveram média de 3,24 (DP=1,06) e os afrodescendentes de 3,33 (DP=1,01), ambas indicando medo da doença, porém não houve diferença significativa entre as etnias [t=1,19; $\mathrm{gl}=688 \mathrm{NS}]$.

\section{Percepção do risco}

Em relação à autopercepção do risco de contrair Aids, houve mais afrodescendentes que disseram não saber se haviam se arriscado a contrair a doença (15,5\% contra 9,2\% dos brancos) e mais brancos que afirmaram não ter se arriscado (76,3\%, contra 69,9\% dos afrodescendentes). Foi verificada associação significativa com etnia $\left[\chi^{2}=6,69 ; g l=2 ; p<0,05\right]$.

Foram comparadas as médias, por etnia, com relação à percepção do risco de pegar Aids, em dois itens escalares de 4 pontos (variando de "muito menos risco" a "muito mais risco"). No primeiro item, a respeito de percepção de risco em relação aos demais brasileiros, os brancos tiveram média de 2,67 ( $D P=0,95)$ e os afrodescendentes, 2,81 ( $D P=1,03)$; não houve diferença estatisticamente significativa por etnias [ $\mathrm{t}=1,45 ; \mathrm{gl}=413$; NS] Em relação ao segundo item, relativo ao risco percebido em relação aos conhecidos, os participantes situaram-se como percebendo risco aproximadamente equivalente; os brancos tiveram média de 1,94 (DP=0,83) e os afrodescendentes apresentaram valor médio de 2,06 $(\mathrm{DP}=\mathrm{de} 0,94)$. A diferença também não foi significativa $[t=1,19 ; \mathrm{gl}=447 ; \mathrm{NS}]$. Observou-se que os participantes pensam que correm mais risco que os brasileiros em geral, mas menos risco do que seus conhecidos.
Foi realizada uma MANOVA $2 \times 2$ com etnia e reconhecimento de ter se arriscado a pegar Aids como variáveis independentes e os dois itens de autopercepção de risco como variáveis dependentes. Não foram encontrados efeitos multivariados significativos para as variáveis "etnia" $[F(2,320)=0,85$; NS; lambda deWilks:0,99], e"ter ou não se arriscado a pegar a Aids $[F(4,640)=0,98$; NS; lambda de Wilks: 0,98] e para a interação entre elas $[F(4,640)=0,66 ; N S ;$ lambda de Wilks:0,99]. A análise dos efeitos univariados não mostrou efeito significativo das variáveis independentes para nenhuma das duas variáveis dependentes investigadas.

\section{Situações de risco}

Por fim, foram realizadas algumas análises sobre a ocorrência de situações diretas ou indiretas de risco diante da transmissão do HIV nos doze meses anteriores. Em termos de proporções de participantes expostos a elas, destacam-se, em ordem decrescente de importância, duas delas: presenciar cenas de violência e ficar bêbado.

Observou-se que mais da metade dos participantes respondeu ter presenciado cenas de violência, indicando que uma quantidade importante dos participantes está exposta ao risco, o que caracteriza sua inserção em contextos de vulnerabilidade social. Não houve diferenças estatisticamente significativas em relação à etnia $\left[\chi^{2}=1,99 ; g \mid=1\right.$; NS $]$.

Quanto ao uso de álcool, 39,3\% dos participantes disseram ter se excedido no consumo de bebidas alcoólicas. Os números mostram uma distribuição homogênea entre os dois grupos, não havendo diferenças estatisticamente significativas em relação à etnia $\left[\chi^{2}=0,10 ; g l=1 ; N S\right]$. Dos estudantes, 89,4\% responderam não ter feito uso de maconha e 90,9\% responderam não ter feito uso de outros tipos de drogas. Dentre os participantes que responderam ter utilizado drogas, as que mais se destacaram foram o cigarro, o ecstasy e a cocaína, correspondendo a respectivamente 2,5\%, 2,4\% e 1,5\% do total de ocorrências.

\section{Conduta protetora, conhecimento sobre a Aids e atitude em relação ao preservativo}

Em relação ao comportamento de proteção contra as DST, 52,5\% disseram que o preservativo foi 
utilizado em todas as relações sexuais que tiveram. Um pouco mais de um quarto da amostra respondeu que utilizou o preservativo em apenas algumas relações e $14,7 \%$ disseram que não costumam utilizar o preservativo. Não há diferença significativa nas proporções conforme a variável etnia $\left[\chi^{2}=2,22 ; \mathrm{gl}=2\right.$; NS].

Ao realizar o cruzamento das variáveis "sexo" e "uso de preservativo na relação sexual", mantendo-se a variável etnia como controle, foi observado que, entre os participantes brancos, a maior proporção de rapazes disse ter usado o preservativo em todas as relações sexuais $(60,2 \%)$, e entre as moças houve equilíbrio entre as que disseram ter usado em algumas relações (42,7\%) e as que declararam ter feito uso em todas (39,5\%). Entre os afrodescendentes, as declarações dos rapazes e das moças foram mais similares; a maioria (63,3\%) dos rapazes disse ter usado preservativo em todas as relações; entre as meninas, predominaram as que disseram ter usado em todas as relações, seguidas pelas que disseram ter usado em algumas relações. A associação entre sexo e uso do preservativo foi estatisticamente significativa para os adolescentes brancos $\left[\chi^{2}=10,96 ; g l=2 ; p<0,01\right]$, mas não para os afrodescendentes $\left[\chi^{2}=5,06 ; g l=2 ; \mathrm{NS}\right]$.

As variáveis "conhecimento sobre a Aids" e "atitude diante do uso do preservativo" correlacionaram-se, mas com índice de pequena magnitude ( $r=0,09$; $p<0,05)$. Foi realizada uma MANOVA $2 \times 3$ com "etnia"e "experiência em relações sexuais" (nunca ter tido relações, ter tido algumas relações ou ter tido várias relações) como variáveis independentes e a "atitude diante do uso do preservativo" e o "resultado no teste de conhecimento sobre a Aids" como variáveis dependentes. Foi encontrado efeito significativo da variável experiência sexual $[F(4,1404)=7,05 ; p<0,001$; lambda de Wilks: 0,96]. Não foram encontrados efeitos multivariados significativos para a variável etnia $[F(2,702)=1,68 ; p ~ N S$; lambda de Wilks: 0,99] e nem para a interação entre etnia e experiência sexual $[F(4,1404)=0,92 ;$ p NS; lambda de Wilks:0,99]. A análise dos efeitos univariados mostrou efeitos significativos da variável experiência sexual na atitude sobre uso de preservativo $[F(2,703)=11,23$; $p<0,001]$, mas não no conhecimento sobre Aids [F(2, 703) $=2,32 ;$ NS]. O teste post-hoc de Tukey diferenciou as pessoas que nunca tiveram relações sexuais das demais $(p<0,05)$. As pessoas sem experiência sexual tiveram atitudes mais favoráveis ao uso do preservativo, isto é, uma média maior na escala $(3,81 ; \mathrm{DP}=0,44)$ que os participantes que tiveram algumas relações sexuais $(M=3,59$ e $D P=0,50)$ ou muitas relações sexuais $(M=3,64$ e DP=0,54).

A partir desses dados, propõem-se dois modelos explicativos que relacionam o sexo seguro, as atitudes sobre o uso do preservativo e a relação amorosa de namoro. Conforme a Figura 2, relacionada aos adolescentes brancos, o uso ou não do preservativo está associado à duas variáveis psicossociais: a experiência afetiva do namoro e a atitude ou o posicionamento do adolescente em relação ao próprio preservativo.

A primeira variável, o namoro, apareceu como um fator complicador para a adoção da conduta do uso sistemático do preservativo, pois os adolescentes que namoram o usam menos do que os que não namo$\operatorname{ram}\left[\chi^{2}=17,49 ; \mathrm{gl}=2 ; p=0,001\right]$; e a segunda como facilitadora deste tipo de conduta preventiva, ou seja: quanto mais favorável ao uso do preservativo, mais sistematicamente ele foi usado nos últimos doze meses $[F(2$, $234)=5,20 ; p<0,01]$. O nível de conhecimento sobre a Aids, para esse grupo de adolescentes, não se relacionou com a atitude sobre o preservativo [ $r=0,07$; NS] e nem com seu uso $[F(2,234)=0,68$; NS $]$.

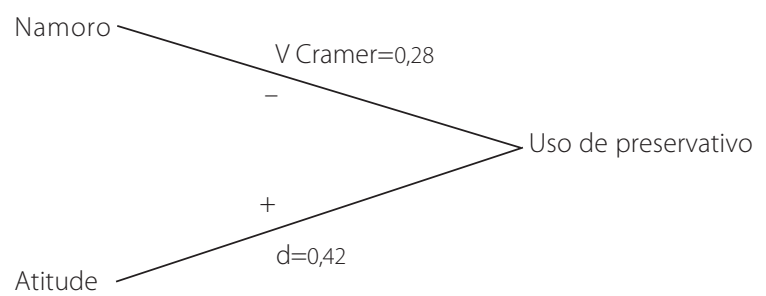

Figura 2. Esquema explicativo para adoção da conduta protetora, relativo aos adolescentes brancos.

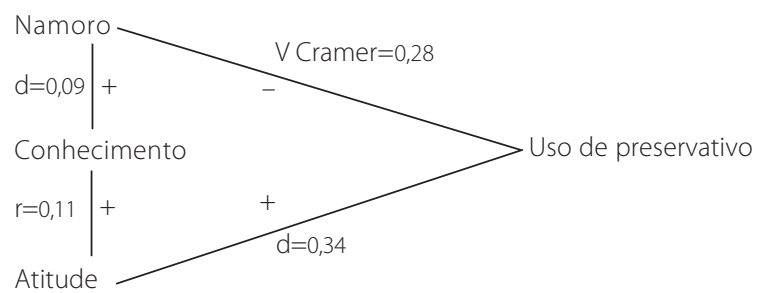

Figura 3. Esquema explicativo para adoção da conduta protetora, relativo aos adolescentes afrodescendentes. 
O mesmo se passa com os adolescentes afrodescendentes (Figura 3): o uso ou não do preservativo está associado ao namoro e a sua atitude em relação ao próprio preservativo. O namoro também apareceu como um fator complicador para a adoção da conduta do uso sistemático do preservativo $\left[\chi^{2}=15,31 ; \mathrm{gl}=2 ; p=0,001\right]$ e a atitude apareceu como fator que prevê esse tipo de conduta preventiva $[F(2,208)=3,13 ; p=0,05]$.

No entanto, entre os afrodescendentes, o nível de conhecimento sobre Aids apresentou-se associado com a atitude sobre o uso do preservativo e com o fato de o adolescente namorar. Um maior conhecimento sobre Aids apresentou-se correlacionado com uma atitude mais favorável sobre o uso do preservativo $[r=0,11$; $p=0,05]$, e os que namoravam tinham um melhor nível de conhecimento sobre a Aids $[F(2,336)=4,25 ; p=0,05]$.

\section{Discussão}

Dentre os participantes da pesquisa, a maioria $(73,7 \%)$ declarou já ter tido relação sexual, diferentemente do que se verificou em estudo anterior, realizado pelo Laboratório de Psicologia Social da Comunicação e Cognição (LACCOS) da Universidade Federal de Santa Catarina (Camargo \& Bertoldo, 2006). No estudo mencionado, que utilizou amostragem por conglomerado das mesmas cidades (Florianópolis, Itajaí e Balneário Camboriú), envolvendo tanto o ensino noturno como o ensino diurno (público e particular), 64,5\% entre os primeiros declararam já terem tido relações sexuais com penetração, e apenas $49,8 \%$ dos alunos do período diurno do ensino público e 42,6\% do diurno particular declararam ter esse tipo de experiência. Assim, os participantes com experiência sexual foram proporcionalmente muito mais numerosos que aqueles da amostra representativa do ensino médio em geral da pesquisa já mencionada.

A amostra utilizada na presente pesquisa, diferente da do estudo anterior, foi intencional. Foram escolhidas escolas situadas na periferia das mesmas cidades e apenas o turno noturno para que se obtivesse um contingente maior de afrodescendentes. Esse dado sobre o comportamento sexual já é um indicador da maior vulnerabilidade dos alunos de escolas da periferia diante das DST e particularmente do HIV.
O nível sociocultural de $80,2 \%$ dos participantes do presente estudo foi classificado como desfavorável, sendo os adolescentes afrodescendentes nessa condição mais numerosos. A proximidade desses adolescentes em relação à Aids e ao HIV não é desprezível: 22,8\% deles disseram que conhecem um amigo nessa situação, e 15,5\% declararam que têm um familiar nessa condição.

O comportamento de "ficar"é bastante presente entre os participantes: mais de dois terços declararam que costumam "ficar". Entre os adolescentes afrodescendentes, esse comportamento é mais frequente do que entre os brancos. O "ficar" apresenta associação estatisticamente significativa com relações sexuais esporádicas, o que foi observado nas duas etnias. As declarações de relações sexuais mais frequentes aconteceram, sobretudo, entre os que disseram não "ficar" (49,5\%), e boa parte em função de namorarem. Entre os "não ficantes", os afrodescendentes são mais numerosos em relação a não terem relação sexual (34,4\% contra 26,2\% dos brancos). Além disso, foram observadas diferenças entre o comportamento de "ficar" e o sexo dos participantes, pois se observou que os rapazes declararam que "ficam" mais do que as moças, numa proporção de $78,2 \%$ para os primeiros e de $58,1 \%$ para as segundas. A prática religiosa dos adolescentes mostrou influenciar o comportamento de "ficar" e sua experiência sexual. Entre os praticantes," fica-se"menos e tem-se menos experiência sexual, e esse padrão é mais acentuado entre os afrodescendentes.

Um dos comportamentos mais associados à conduta arriscada é o de namorar, pois tanto para afrodescendentes quanto para brancos, esse é um fator que diminui muito o uso de preservativo. Vários estudos anteriores (Giacomozzi, 2005; Giacomozzi \& Camargo, 2004; Oltramari \& Camargo, 2004) demonstraram que o fato de viver no momento uma relação estável diminui o uso sistemático do preservativo. Além disso, os usos de outros métodos anticoncepcionais, como a pílula, também contribuem para a prática do sexo sem proteção. Porém esse comportamento (o de namorar) apresentou-se de modo diverso para as duas etnias. A proporção de participantes que declarou namorar é maior entre brancos do que entre afrodescendentes ea duração do namoro também é maior entre os primeiros. Esse dado poderia explicar uma parte da vulnerabilidade dos brancos diante das DST e da Aids, pois o compor- 
tamento de namorar foi identificado como um comportamento complicador para a adoção de práticas preventivas. Por outro lado, como os afrodescendentes namoram menos, não apresentariam essa vulnerabilidade, porém possuem relações mais esporádicas, nas quais o preservativo nem sempre é utilizado.

No que diz respeito à dimensão informativa, apesar de não serem estatisticamente significativas, houve diferenças nos escores obtidos no subteste usado para medir conhecimento sobre HIV/Aids. Os participantes brancos apresentaram mais acertos do que os afrodescendentes, no entanto as duas etnias obtiveram média inferior ao ponto de corte, o que significa que ambas não apresentam um conhecimento científico adequado sobre a matéria. Calazans (2005) observou que jovens com maior escolaridade e renda familiar referem informações mais corretas sobre Aids. Camargo e Bertoldo (2006) reportaram que adolescentes que dispunham de informação adequada sobre Aids apresentavam uma atitude mais positiva sobre o uso sistemático do preservativo.

Mas se os acertos no subteste de conhecimento sobre HIV/Aids indicaram que os participantes de ambas as etnias apresentam problemas sobre isso, os afrodescendentes, que comparativamente tem um menor conhecimento, são os que apresentaram uma autopercepção mais realista sobre seu conhecimento, já que somente $42,9 \%$ se acham bem informados sobre a matéria, enquanto entre os brancos foram 50,0\% que perceberam dessa mesma forma. Quanto à fonte de informação sobre Aids, a escola, a televisão e a família aparecem, por ordem, como principais para os adolescentes participantes desta pesquisa. A atitude das duas etnias foi favorável ao uso do preservativo (respectivamente 3,69 e 3,65 numa escala onde o ponto médio é 3), porém se observou que as moças têm uma atitude mais favorável ao uso do preservativo que os rapazes. Esses dados confirmam os encontrados em uma etapa anterior desta pesquisa (Camargo, Giacomozzi, Wachelke \& Aguiar, 2007), quando, nas entrevistas, as meninas declararam considerar importante o uso do preservativo como método contraceptivo e de proteção diante das DST e Aids. Apesar disso, elas encontravam barreiras na negociação do seu uso com seus parceiros. Os dados sobre a atitude em relação ao uso do preservativo mostram uma maior diferença entre os sexos do que entre as etnias dos participantes.
Os adolescentes relataram sentir medo em relação à Aids (respectivamente 3,24 para os brancos e 3,33 para afrodescendentes) numa escala de 1 a 4. Esse sentimento de medo pode favorecer a adoção de condutas preventivas, mas dependendo da sua intensidade, pode ser prejudicial (McGuire, 1976). Apesar de terem uma atitude favorável ao uso do preservativo, os participantes não se sentem particularmente vulneráveis diante da doença: mesmo percebendo que correm mais risco que a maioria dos brasileiros, acham que correm menos risco que seus conhecidos.

O uso abusivo de drogas neste estudo não foi menos importante: 39,3\% dos participantes declararam terem ficado bêbados nos últimos doze meses. Essa proporção é superior às encontradas no estudo de Camargo e Bertoldo (2006), onde 31,5\% dos alunos de escolas particulares, $24,1 \%$ dos alunos de escola pública no diurno e $23,4 \%$ no noturno tiveram essa mesma experiência. Outro dado importante é a associação estatisticamente significativa entre a declaração do uso de maconha e o uso de outras drogas. O uso abusivo de drogas consiste num fator indireto de risco em relação à infecção pelo HIV, pois ele traz dificuldades para o adolescente adotar uma conduta preventiva diante do vírus. O uso de drogas também surgiu como fator presente na vida dos entrevistados na primeira etapa desta pesquisa, confirmando a proximidade e a exposição dessa população a essa realidade.

Mais da metade dos participantes $(63,2 \%)$ declarou ter presenciado cenas de violência nos últimos doze meses. Esse dado parece fazer sentido diante dos achados de Santos, Santos e Borges (2005), nos quais 55,0\% dos adolescentes entrevistados (com mesma faixa etária dos participantes da presente pesquisa) preocupavam-se com sua segurança, e quase metade dos adolescentes entrevistados (46,0\%) disse já ter experimentado tal situação. Ainda cabe observar que essa realidade foi mais vivenciada por adolescentes negros do que brancos, pois 52,0\% dos negros disseram ter perdido alguém de forma violenta, enquanto isso aconteceu a 49,0\% dos pardos e $42,0 \%$ dos adolescentes brancos.

Assim, a situação sociocultural desfavorável, juntamente com as diferenças de comportamento entre os sexos e a faixa etária, são fatores importantes para a vulnerabilidade dos adolescentes de escolas de periferia. Provavelmente, a associação da etnia à vulnerabilidade 
ao HIV/Aids se deve ao fato de ela, no Brasil, de antemão, estar associada a uma situação sociocultural desvantajosa, e à discriminação racial dos afrodescendentes no cotidiano das relações interpessoais, das relações escolares, das relações de trabalho, entre outras.

\section{Considerações Finais}

Os resultados desta pesquisa mostram que, de forma geral, a maioria dos participantes, afrodescendentes ou não, estão vulneráveis ao HIV e à Aids em função da importante presença do sexo desprotegido e da sua associação com as relações amorosas (principalmente o namoro), da proximidade com pessoas infectadas pelo HIV, da exposição à violência, do uso de drogas e do conhecimento insuficiente sobre a doença. A composição de tais fatores, associada à falta de acesso à informação sistemática e de qualidade e a serviços de saúde eficientes, como ocorre com a população mais carente (cuja maioria é da raça negra), diminui as possibilidades de adoção de comportamentos preventivos. Essas desigualdades sociais e raciais são muito antigas em nossa sociedade e estão no cerne da vulnerabilidade específica dessa população ao HIV/Aids.

Os dados mostram, portanto, que a vulnerabilidade dos afrodescendentes não está diretamente associada com sua etnia, mas sim às dificuldades econômicas, sociais e culturais que a população de camadas populares - de maioria predominante da raça negra enfrenta no Brasil. Nas palavras de Santos, Santos e Borges (2005, p.292):"A composição social do Brasil tem a base bastante larga e o ápice estreito. Há, porém, uma característica ainda mais marcante: as camadas sociais vão embranquecendo na medida em que sobem na pirâmide social. Assim, quando se fala das pessoas que vivem na base da pirâmide social, logo se identifica a pobreza, e quando se fala de pobreza no Brasil, está-se falando principalmente da população negra e da discriminação racial". Segundo os autores anteriormente citados, para uma melhor compreensão da questão dos adolescentes negros no Brasil, é importante que se leve em conta as relações sociais inter-raciais que esses adolescentes estabelecem, marcadas por grande desigualdade social do país, que se somam à discriminação racial.
Além disso, é necessário que se amplie o acesso da população jovem de camadas populares aos serviços, insumos e informações em HIV/Aids. Porém tais informações e insumos não devem se restringir aos ambientes escolares e de saúde, visto que muitos desses jovens não frequentam escolas ou serviços de saúde. Outro aspecto importante a ser trabalhado é o estabelecimento de políticas de prevenção, assistência e direitos humanos para a população de camadas populares, bem como o fomento à pesquisa e ao conhecimento sobre Aids, preconceito, pobreza, equidade, racismo e suas relações com a vulnerabilização ao HIV/Aids.

\section{Referências}

Ayres, J. R. C. M., Júnior, F., \& Calazans, G. J. (1997). Saúde reprodutiva em tempos de AIDS (II Seminário), AIDS, vulnerabilidade e prevenção. Rio de Janeiro: ABIA.

Ayres, J. R. C. M.; França Júnior, I; Calazans, G. J., \& Salletti Filho, H. C. (1999). Vulnerabilidade e prevenção em tempos de AIDS. In M.R. Barbosa \& R. Parker (Orgs.), Sexualidade pelo avesso: direitos, identidades e poder (pp.49-72). Rio de Janeiro: IMS/UERJ.

Bastos, F.I., Telles, P.R., Castilho, E., \& Barcellos, C. (1999). A epidemia de AIDS no Brasil. In M.C.S. Minayo (Org.), Os muitos brasis: saúde e população na década de 80 (2a ed., pp.245-268). São Paulo: Hucitec.

Batista, L.E. (2005). Masculinidade, raça/cor e saúde. Rio de Janeiro. Ciência \& Saúde Coletiva, 10 (1), 71-80.

Brasil. Ministério da Saúde. (2006/2007). Boletim Epidemiológico DST/AIDS, 4(1). Recuperado em novembro, 2008, disponível em www.aids.gov.br/data/documents

Brasil. Ministério da Saúde. (2003). Políticas e diretrizes de prevenção das DST/AIDS. Brasília: Ministério da Saúde.

Calazans, G. (2005). Os jovens falam sobre sua sexualidade e saúde reprodutiva: elementos para a reflexão. In $\mathrm{H}$. Wendel \& P. Branco (Orgs.), Retratos dajuventude brasileira: análise de uma pesquisa nacional (pp.215-242). São Paulo: Fundação Perseu Abrano.

Camargo, B. V. (1997). Communication et prévention du sida. Études sur le rapport entre l'information télévisuelle, les représentations sociales et la pratique préventive chez les jeunes lycéens. Tese de doutorado não-publicada, Ecole des Hautes Etudes en Sciences Sociales, Paris, França.

Camargo, B. V. Bárbara, A., \& Bertoldo, R. (2005). Um instrumento de medida da dimensão informativa da representação social da AIDS. In IV Jornada Internacional ell Conferência Brasileira sobre Representações Sociais. João Pessoa. (Vol.1, pp.1385-1395).

Camargo, B. V., \& Bertoldo, R. B. (2006). Comparação da vulnerabilidade de estudantes da escola pública e particular em relação ao HIV. Estudos de Psicologia (Campinas), 23 (4), 369-379. 
Camargo, B., Botelho, L., \& Souza, E. (2001). AIDS, sexualidade eatitudes sobre a proteção frente ao HIV: um estudo descritivo com adolescentes do nível médio da rede de ensino (Florianópolis, Itajaí e Balneário Camburiú). (Relatório Técnico de Pesquisa). Florianópolis: UFSC.

Camargo, B. V., Giacomozzi, A.I., Wachelke, J.F.R., \& Aguiar, A. (2007). Estudo exploratório sobre etnia e vulnerabilidade frente a AIDS. V Jornada Internacional sobre Representações Sociais. Brasília.

Cochran, S. D., \& Mays, M. (1989). Women and AIDS-related: concerns roles for psychologists in helping the worried well. American Psychologist, 44 (3), 529-535.

Fishbein, M., \& Ajzen, I. (1975). Belief, attitude, intention and behavior: an introduction to theory and research. Rfeading, MA: Addison-Wesley.

Giacomozzi, A. I., \& Camargo, B. V. (2004). Eu confio no meu marido: estudo da representação social de mulheres com parceiro fixo sobre a prevenção da AIDS. Psicologia, $6(4), 31-44$.

Giacomozzi, A. I. (2005). Casamento e Aids: uma questão de confiança. São Paulo: Mackenzie.

Keels, C. L. (2005). Saving a generation: North Carolina public health department partners with state's Black colleges to raise awareness about HIV/aids. Black Issues in Higher Education, 22 (3), 34-35.

Leite, I. B. (1996). Descendentes de africanos em SC: invisibilidade histórica e segregação. In I.B. Leite (Org.), Negros no sul do Brasil:invisibilidadee territorialidade (pp.3353). Florianópolis: Letras Contemporâneas.
Leite, I. B. (1988). Os sentidos da cor e impurezas do nome. Florianópolis: USSC.

Lopes, F., Buchalla, C. M., \& Ayres, M. C. R. J. (2007). Mulheres negras e não negras e vulnerabilidade ao HIV/AIDS no estado de São Paulo, São Paulo. Revista de Saúde Pública, 41 (supl 2), 39-46.

McGuire, W. (1976). Persuasão. In G.A. Miller (Org.) Linguagem, psicologia e comunicação (pp.258-271). São Paulo: Cultrix.

Moscovici, S. (2004). Representações sociais: investigações em psicologia social (2a ed.) Rio de Janeiro: Vozes.

Oltramari, L. C., \& Camargo, B.V. (2004). Representação social de mulheres profissionais do sexo sobre a AIDS, Natal. Estudos de Psicologia, 9 (2), 317-324.

Santos,G., Santos, M. J. P., \& Borges, R. (2005) A juventude negra. In H. Wendel \& P. Branco (Orgs.), Retratos da juventude brasileira: análise de uma pesquisa nacional (pp.291-302). São Paulo: Fundação Perseu Abrano.

Stroebe, W., \& Stroebe, M. (1995). Psicologia social e saúde. Lisboa: Instituto Piaget.

Vala, J. (2000). Representações sociais e psicologia social do conhecimento cotidiano. In J. Vala \& B. Monteiro (Orgs.), Psicologia social (pp.457-502). Lisboa: Fundação Calouste Gulbenkian.

Recebido em: 14/2/2008

Versão final reapresentada em: 12/7/2010

Aprovado em: 17/8/2010 